\title{
COHOMOLOGY AND DEFORMATIONS IN GRADED LIE ALGEBRAS ${ }^{1}$
}

\author{
ALBERT NIJENHUIS AND R. W. RICHARDSON, JR.
}

Introduction. In an address to the Society in 1962, one of the authors gave an outline of the similarities between the deformations of complex-analytic structures on compact manifolds on one hand, and the deformations of associative algebras on the other. The first theory had been stimulated in 1957 by a paper [7] by NijenhuisFrölicher and extensively developed in a series of papers by KodairaSpencer, Kodaira-Spencer-Nirenberg and Kuranishi; the second had just been initiated by Gerstenhaber [9]. While fine details were not available at that time, it seemed that graded Lie algebras were the common core of both theories. In particular, in both cases, the set of deformed structures is represented by the set of solutions of a certain deformation equation in graded Lie algebras. This observation was further elaborated in a Research Announcement [16] of the authors, in which the concept of algebraic graded Lie algebra was carefully defined, and in which applications to deformations of Lie algebras and to representations, extensions and homomorphisms of algebras were indicated.

The present paper gives a detailed discussion of the deformation equation in graded Lie algebras whose summands are finite-dimensional. The paper starts with a general discussion of graded Lie algebras including the case of characteristic 2 , and leads to a general deformation theorem, which is the precise analogue of Kuranishi's local completeness theorem for complex analytic structures [13]. (A more recent proof of this theorem [14] uses methods closely related to those indicated in [16].)

The basic deformation theorems presented are 16.2, 18.1, 20.3, 22.1 and 23.4. The following is an outline in which a few of the more technical details have been deleted. We consider a graded Lie algebra (cf. 3.1) $E=\bigoplus_{n=0}^{\infty} E^{n}$ in which each $E^{n}$ is finite-dimensional, and with

Based on an address (entitled Derivations and structures) by Professor Nijenhuis delivered before the Vancouver meeting of the Society on August 28, 1962 by invitation of the Committee to Select Hour Speakers for Annual and Summer Meetings; received by the editors August 27, 1965.

1 Research supported by an Office of Naval Research contract at the University of Washington, a Fulbright grant at the University of Amsterdam, an N.S.F. Postdoctoral Fellowship at the Institute for Advanced Study and the Mathematical Institute at Oxford, and N.S.F. Grant GP 1905 at the University of Pennsylvania. 
which is associated a group $G$ of automorphisms (cf. 9.1, 10.1), whose Lie algebra is $E^{0}$ and acts by the adjoint representation of $E^{0}$ on $E$. Furthermore, there is given a derivation $D$ on $E$ which raises degrees by 1 , and which satisfies $D^{2}=0$. (One may take $D=0$ and still get meaningful results.) The equation

$$
D a+\frac{1}{2}[a, a]=0 \quad\left(a \in E^{1}\right)
$$

is called the deformation equation; let $M_{0}$ be the algebraic set of all solutions of this equation. Then $M_{0}$ is stable under an appropriate action of $G$; points of $M_{0}$ on the same orbit are called equivalent. (In specific applications $M_{0}$ will be the set of all associative structures on a given vector space, the set of all representations of a given algebra on a given vector space, the set of all homomorphisms of one algebra into another, etc. Equivalent points correspond to isomorphic associative algebras, equivalent representations, etc.)

A point $a \in M_{0}$ is rigid if its $G$-orbit is a neighborhood of $a$ in $M_{0}$; we set $D_{a} b=D b+[a, b]$ for $b \in E$; consequently $D_{a}^{2}=0$, and $E$ can be considered as a cochain complex with coboundary operator $D_{a}$. Then the space of coboundaries $B^{1}\left(E, D_{a}\right)$ is the tangent space to the $G$ orbit of $a$ at $a$; the space of cocycles $Z^{1}\left(E, D_{a}\right)$ the tangent space at $a$ to $M_{0}$. The rigidity theorem (cf. 16.2, 22.1) states that if $H^{1}\left(E, D_{a}\right)$ $=0$, then $a$ is rigid. (For associative and Lie algebras $A$ this condition translates into $H^{2}(A, A)=0$, for the Hochschild resp. ChevalleyEilenberg cohomologies.)

A connected subset $F$ of $M_{0}$ containing $a$ is locally complete at $a$ if, for every neighborhood $U$ of $a$, the $G$-orbit of the $a$-component of $U \cap F$ is a neighborhood of $a$ in $M_{0}$. It is proved (cf. 20.3, 23.4), that the so-called Kuranishi families are locally complete. The general description of Kuranishi families is moderately involved; when the base field is the real or complex numbers it is a subvariety of $M_{0}$ analytically parametrizable by a subvariety of $H^{1}\left(E, D_{a}\right)$ which in its turn is the set of zeros of an analytic map of $H^{1}\left(E, D_{a}\right)$ into $H^{2}\left(E, D_{a}\right)$. As a corollary, $a$ is a simple point of $M_{0}$ if $H^{2}\left(E, D_{a}\right)=0$. (For associative and Lie algebras the groups $H^{n}\left(E, D_{a}\right)$ are the corresponding $H^{n+1}(A, A)$ of Hochschild and Chevalley-Eilenberg.)

The following are typical applications of the general theorems. Their proofs are just the constructions of specific graded Lie algebras relevant to the problems considered. For the associative algebras this construction is given in [8], for the Lie algebras in [16]; others are to be given in a forthcoming paper. Some general comments are given in $\$ 3$.

(i) Let $M$ be the algebraic set of all Lie algebra multiplications on 
a finite-dimensional vector space $V$ over an algebraically closed field, and let $G=\mathrm{GL}(V)$. If $\mu \in M$ is such that the Lie algebra $L=(V, \mu)$ satisfies $H^{2}(L, L)=0$, then $\mu$ is rigid (its $G$-orbit is Zariski-open in $M)$. There are only finitely many isomorphism classes of Lie algebras $L=(V, \mu)$ with $H^{2}(L, L)=0$.

(ii) Let $L$ be a finite-dimensional Lie algebra and $W$ a finitedimensional vector space over an algebraically closed field. Let $M$ denote the algebraic set of all representations of $L$ on $W$; i.e. each $\rho \in M$ is a homomorphism $\rho: L \rightarrow \operatorname{End}(W)$; it makes $\operatorname{End}(W)$ into an $L$-module. If $\rho \in M$ is such that $H^{1}(L, \operatorname{End}(W))=0$, then the set of representations which are equivalent to $\rho$ is a Zariski-open subset of $M$. There are only finitely many equivalence classes of $\rho$ for which $H^{1}(L, \operatorname{End}(W))=0$.

The authors are thanking M. Gerstenhaber and P. Griffiths for several helpful discussions concerning this material.

0 . Notation. The five major parts of the paper are denoted $\S \S 1-5$. The sections, 0 through 24 , are numbered independently.

$\boldsymbol{Z}$ denotes the ring of rational integers. $\boldsymbol{Z}_{n}$ denotes the quotient ring $\boldsymbol{Z} / n \boldsymbol{Z}$. $\boldsymbol{R}$ (resp. C) denotes the field of real (resp. complex) numbers. Fields are to be commutative.

We shall use the notation $V=\bigoplus_{\alpha \in \Delta} V^{\alpha}$ for both internal and external direct sums of vector spaces. In particular, we shall consider $V^{\alpha}$ as a subspace of $\oplus_{\alpha} V^{\alpha}$ without further comment.

We denote by $\mathrm{GL}(V)$ the group of all automorphisms of the vector space $V$ (ignoring any additional structure $V$ might have); $\mathfrak{g l}(V)$ denotes the Lie algebra of all endomorphisms of $V$.

Algebraic groups will be taken in the sense of Chevalley's book [2]. That is, an algebraic group is a subgroup $G$ of the group GL( $V)$ (for some finite dimensional vector space $V$ ) which is of the form $G=\mathrm{GL}(V) \cap A$, where $A$ is an algebraic subset of the vector space of all endomorphisms of $V$.

\section{\$1. GRaded Lie algebras}

In this part we discuss the basic properties of graded Lie algebras. We have chosen to give a rather detailed treatment, first, because there is no such account in the literature, and secondly, in order to prepare the way for future applications of the theory. In particular, we discuss graded Lie algebras graded by an arbitrary commutative group with parity. In $\S \S 9$ and 10 we introduce the notions of analytic and algebraic graded Lie algebras, which will be basic for the deformation theorems of $\S \S 4$ and 5 . 
1. Graded algebras. Let $\Delta$ be a commutative group. A graded vector space of type $\Delta$ is a vector space $V$ together with a family $\left\{V^{\alpha}\right\}_{\alpha \in \Delta}$ of subspaces of $V$, indexed by $\Delta$, such that $V$ is the direct sum of the family $\left\{V^{\alpha}\right\}$ of subspaces. The elements of $V^{\alpha}$ are called homogeneous of degree $\alpha$. A subspace $W$ of $V$ is a graded subspace if $W$ is the sum (necessarily direct) of the family of subspaces $\left\{W \cap V^{\alpha}\right\}$; if this is so, then $W$ itself is a graded vector space of type $\Delta$. Let $W$ be a graded subspace of $V$ and let $\pi: V \rightarrow V / W$ denote the canonical projection. Then the family $\left\{\pi\left(V^{\alpha}\right)\right\}$ defines a graded vector space structure on $V / W$.

If $V=\oplus_{\alpha \in \Delta} V^{\alpha}$ and $W=\oplus_{\alpha \in \Delta} W^{\alpha}$ are graded vector spaces of type $\Delta$, we define a structure of graded vector space of type $\Delta$ on the direct sum $U=V \oplus W$ by setting $U^{\alpha}=V^{\alpha} \oplus W^{\alpha}$ for every $\alpha \in \Delta$.

A linear map $f$ of a graded vector space $V=\oplus_{\alpha \in \Delta} V^{\alpha}$ into a graded vector space $W=\bigoplus_{\alpha \in \Delta} W^{\alpha}$ is homogeneous of degree $\beta \in \Delta$ if, for every $\alpha \in \Delta$, we have $f\left(V^{\alpha}\right) \subset W^{\alpha+\beta}$; when this is so, the kernel (resp. image) of $f$ is a graded subspace of $V$ (resp. $W$ ). Let $H$ denote the vector space of all linear maps of $V$ into $W$ and, for each $\alpha \in \Delta$, let $H^{\alpha}$ be the subspace of $H$ consisting of all homogeneous linear maps of degree $\alpha$. We define $\operatorname{Hom}(V, W)$ to be the sum of the family $\left\{H^{\alpha}\right\}_{\alpha \in \Delta}$ of subspaces of $H$. It is easily verified that this sum is direct and thus defines on $\operatorname{Hom}(V, W)$ the structure of a graded vector space; we denote $H^{\alpha}$ by $\operatorname{Hom}^{\alpha}(V, W)$.

A graded algebra of type $\Delta$ over a field $K$ is a graded vector space $A=\oplus_{\alpha \in \Delta} A^{\alpha}$ over $K$ which is given an algebra structure compatible with its graded structure, i.e. a bilinear map $(a, b) \rightarrow a b$ of $A \times A$ into $A$ such that $A^{\alpha} A^{\beta} \subset A^{\alpha+\beta}$ for every $\alpha, \beta \in \Delta$. (If $B$ and $C$ are subspaces of $A$, we denote by $B C$ the subspace of $A$ spanned by the set of products $\{b c \mid b \in B, c \in C\}$.) A graded subalgebra of a graded algebra $A$ is a graded subspace $B$ of $A$ which is closed under products, i.e. $B B C B$. A graded ideal of a graded algebra $A$ is a graded subspace $C$ of $A$ such that $A C \subset C$ and $C A \subset C$. A homomorphism of a graded algebra $A$ of type $\Delta$ into a graded algebra $B$ of type $\Delta$ is a homogeneous linear map $f$ of degree zero of $A$ into $B$ such that $f(a b)$ $=f(a) f(b)$ for every $a, b \in A$. Let $A$ be a graded algebra, let $C$ be a graded ideal in $A$, and let $\pi: A \rightarrow A / C$ denote the canonical projection. Then there exists a unique structure of graded algebra on $A / C$ such that $\pi$ is a homomorphism of graded algebras.

The concept of associativity for graded algebras is the usual one: $(a b) c=a(b c)$.

Let $V=\oplus_{\alpha} V^{\alpha}$ be a graded vector space. If $f \in \operatorname{Hom}^{\alpha}(V, V)$ and $g \in \operatorname{Hom}^{\beta}(V, V)$, then $f \circ g \in \operatorname{Hom}^{\alpha+\beta}(V, V)$. It follows easily from this observation that if $f \in \operatorname{Hom}(V, V)$ and $g \in \operatorname{Hom}(V, V)$, then 
$f \circ g \in \operatorname{Hom}(V, V)$. Thus the graded vector space $\operatorname{Hom}(V, V)$, together with the bilinear map $(f, g) \rightarrow f \circ g$ is a graded associative algebra; we denote it by $\operatorname{End}(V)$.

2. Commutative and anticommutative graded algebras. A commutative group with parity is a commutative group $\Delta$, together with a homomorphism $\phi$ of $\Delta$ into the commutative group $\boldsymbol{Z}_{2}$. The elements of $\phi^{-1}(0)$ are called even, those of $\phi^{-1}(1)$ are called odd.

Let $K$ be a commutative ring with identity. The map $n \rightarrow(-1)^{n}$ is a homomorphism of the commutative group $\boldsymbol{Z}$ into the (multiplicative) group $K^{*}$ of units of $K$; the kernel of this map includes $2 Z$. Thus there is an induced homomorphism of $\boldsymbol{Z}_{2}$ into $K^{*}$. The image of an element $a \in \boldsymbol{Z}_{2}$ under this homomorphism will be denoted $(-1)^{a}$.

Definition 2.1. Let $A$ be a graded algebra over a field $K$ of characteristic $\neq 2$ graded by a commutative group with parity $\Delta$ (with parity map $\phi$ ). Then $A$ is a commutative (resp. anticommutative) graded algebra if formula (i) (resp. (ii)) below holds for every pair of homogeneous elements, $a \in A^{\alpha}, b \in A^{\beta}$ :

(i) $a b=(-1)^{\phi(\alpha) \phi(\beta)} b a$;

(ii) $a b=-(-1)^{\phi(\alpha) \phi(\beta)} b a$.

The classical notions of commutativity and anticommutativity are obtained by taking $\phi$ trivial: $\phi(\Delta)=\{0\}$.

In most examples the grading group $\Delta$ will be the additive group $\boldsymbol{Z}$ of integers and $\boldsymbol{\phi}$ will be the canonical projection $\boldsymbol{Z} \rightarrow \boldsymbol{Z} / 2 \boldsymbol{Z}$. We then have, for $\alpha, \beta \in \Delta,(-1)^{\phi(\alpha) \phi(\beta)}=(-1)^{\alpha \beta}$. By abuse of notation we shall write $(-1)^{\alpha \beta}$ for general $\Delta$ and $\phi$ also. Thus $(-1)^{\alpha \beta}=-1$ if both $\alpha$ and $\beta$ are odd, otherwise $(-1)^{\alpha \beta}=1$.

We always consider $\boldsymbol{Z}$ as a commutative group with parity map the natural projection $\boldsymbol{Z} \rightarrow \boldsymbol{Z} / 2 \boldsymbol{Z}$.

3. Graded Lie algebras. For the rest of $\S \S 1$ and 2, unless explicitly stated otherwise, all graded vector spaces and graded algebras will be of type $\Delta$, where $\Delta$ is a commutative group with parity. Explicit reference to $\Delta$ will of ten be suppressed.

A graded Lie algebra is an anticommutative graded algebra which satisfies a graded version of the classical Jacobi identity. In detail:

Definition 3.1. A graded Lie algebra of type $\Delta$ over a field $K$ of characteristic $\neq 2$ is a graded vector space $E=\bigoplus_{\alpha \in \Delta} E^{\alpha}$ over $K$ together with a bilinear map $(a, b) \rightarrow[a, b]$ of $E \times E$ into $E$ which satisfies the following conditions:

GLA1. $\left[E^{\alpha}, E^{\beta}\right] \subset E^{\alpha+\beta}$ for all $\alpha, \beta \in \Delta$;

GLA2. If $a \in E^{\alpha}$ and $b \in E^{\beta}$, then $[a, b]=-(-1)^{\alpha \beta}[b, a]$;

GLA3. If $a \in E^{\alpha}, b \in E^{\beta}$ and $c \in E^{\gamma}$, then 


$$
(-1)^{\alpha \gamma}[[a, b], c]+(-1)^{\beta \alpha}[[b, c], a]+(-1)^{\gamma \beta}[[c, a], b]=0 .
$$

When $\operatorname{char} K=3$, this is supplemented by the condition: if $a$ is a homogeneous element of odd degree, then $[[a, a], a]=0$.

The identity given in GLA3 is called the Jacobi identity. It can be written in the following equivalent forms:

$$
\begin{gathered}
{[a,[b, c]]=[[a, b], c]+(-1)^{\alpha \beta}[b,[a, c]]} \\
{[a,[b, c]]-(-1)^{\alpha \beta}[b,[a, c]]=[[a, b], c]} \\
(-1)^{\alpha \gamma}[a,[b, c]]+(-1)^{\beta \alpha}[b,[a, c]]+(-1)^{\gamma \beta}[c,[a, b]]=0
\end{gathered}
$$

We shall discuss the concepts of commutativity, anticommutativity, and graded Lie algebras over fields of characteristic 2 separately in $\$ 2$.

Remarks. 1. A Lie algebra may be considered as a graded Lie algebra all of whose elements are of degree 0 .

2. The center of a graded Lie algebra $E$ is the graded ideal $\{a \in E \mid[a, x]=0$ for all $x \in E\}$.

3. The subalgebra $E^{0}$ of $E$ is an (ungraded) Lie algebra. More generally, $\oplus_{\alpha \text { even }} E^{\alpha}$ has the structure of (ungraded) Lie algebra, denoted $\varepsilon^{0}$. Setting $\mathcal{E}^{1}=\oplus_{\alpha \text { odd }} E^{\alpha}$, we see that $\mathcal{E}^{0} \oplus \mathcal{E}^{1}$ is a graded Lie algebra of type $\boldsymbol{Z}_{2}$.

4. Graded Lie algebras of commutators. The commutator product defines on an (ungraded) associative algebra the structure of a Lie algebra. A similar result holds for graded associative algebras, provided we take a graded commutator product.

Proposition 4.1. Let $A=\oplus_{\alpha} A^{\alpha}$ be a graded associative algebra. The underlying graded vector space of $A$, with the product determined by $[a, b]=a b-(-1)^{\alpha \beta} b a$ for $a \in A^{\alpha}, b \in A^{\beta}$, is then a graded Lie algebra.

The proofs of GLA1 and GLA2 are immediate. GLA3 is verified simply by a write-out.

As a particular example let $V=\oplus_{\alpha} V^{\alpha}$ be a graded vector space. Then we have seen that $\operatorname{End}(V)$ has a natural structure of graded associative algebra. The graded Lie algebra derived from $\operatorname{End}(V)$ by the process above is denoted by $\mathfrak{g l}(V)=\oplus_{\alpha} \mathrm{gl}^{\alpha}(V)$. If $f$ and $g$ are homogeneous endomorphisms of $V$ of odd degree, then $[f, g]=f \circ g$ $+g \circ f$; so $[f, g]$ is not in this case the usual commutator of endomorphisms. We note that the Lie algebra $\mathfrak{g l}^{0}(V)$ is canonically isomorphic to the direct product of the family of Lie algebras $\left\{\operatorname{gl}\left(V^{\alpha}\right)\right\}_{\alpha}$.

The construction of Proposition 4.1 also works for graded algebras satisfying a somewhat more general law than associativity. 
Proposition 4.2. Let $A$ be a graded algebra whose product satisfies one of the following commutative-associative laws: if $a \in A^{\alpha}, b \in A^{\beta}$ and $c \in A^{\gamma}$, then

$$
\begin{aligned}
& a(b c)-(a b) c=(-1)^{\beta \gamma}(a(c b)-(a c) b) ; \\
& a(b c)-(a b) c=(-1)^{\alpha \beta}(b(a c)-(b a) c) ; \\
& a(b c)-(a b) c=(-1)^{\alpha \beta+\beta \gamma+\gamma \alpha}(c(b a)-(c b) a) .
\end{aligned}
$$

The underlying vector space of $A$, with the product determined by $[a, b]$ $=a b-(-1)^{\alpha \beta} b a$ for $a \in A^{\alpha}, b \in A^{\beta}$ is then a graded Lie algebra.

Proof. As GLA1, 2 are obvious, we need prove only GLA3. We give the proof only for the case of (4.3). The other two cases are similar. We rewrite (4.3) as follows:

$$
(-1)^{\alpha \gamma}(a b) c-(-1)^{(\alpha+\beta) \gamma}(a c) b=(-1)^{\alpha \gamma} a[b, c] .
$$

Now we permute $a, b, c$ (and also $\alpha, \beta, \gamma$ ) cyclically. This gives two more identities:

$$
\begin{aligned}
& (-1)^{\beta \alpha}(b c) a-(-1)^{(\beta+\gamma) \alpha}(b a) c=(-1)^{\beta \alpha} b[c, a], \\
& (-1)^{\gamma \beta}(c a) b-(-1)^{(\gamma+\alpha) \beta}(c b) a=(-1)^{\gamma \beta} c[a, b] .
\end{aligned}
$$

Addition of the three identities gives

$$
\begin{aligned}
& (-1)^{\gamma \alpha}[a, b] c+(-1)^{\alpha \beta}[b, c] a+(-1)^{\beta \gamma}[c, a] b \\
& \quad=(-1)^{\alpha \gamma} a[b, c]+(-1)^{\beta \alpha} b[c, a]+(-1)^{\gamma \beta} c[a, b] .
\end{aligned}
$$

Collecting terms from both sides we find GLA3. If char $K=3$, we again take (4.3) and set $a=b=c \in A^{\alpha}$, with $\alpha$ odd. Since $[a, a]=2 a a$ this gives $a[a, a]-[a, a] a=0$; that is, $[[a, a], a]=0$.

The graded Lie algebras which are used in studying deformations of Lie algebras and associative algebras are obtained from graded algebras which satisfy the commutative-associative law (4.3).

5. Graded Lie algebras and derivations. Let $A=\oplus_{\alpha} A^{\alpha}$ be a graded algebra and let $D: A \rightarrow A$ be a homogeneous linear map of degree $\alpha$. Then $D$ is a derivation of degree $\alpha$ if

$$
D(a b)=(D a) b+(-1)^{\alpha \beta} a(D b) \quad \text { for } a \in A^{\beta}, b \in A .
$$

The set $D^{\alpha}(A)$ of all derivations of degree $\alpha$ of $A$ is a subspace of $\operatorname{Hom}^{\alpha}(A, A)$. Let $D(A)$ denote the sum of the family $\left\{D^{\alpha}(A)\right\}_{\alpha}$ of subspaces of $\operatorname{Hom}(A, A) ; D(A)$ is a graded subspace of $\operatorname{Hom}(A, A)$, or equivalently, of $\mathfrak{g l}(A)$.

Proposition 5.1. $\mathfrak{D}(A)$ is a graded subalgebra of the graded Lie algebra $\mathfrak{g l}(A)$. 
Proof. It must be shown that the product $\left[D, D^{\prime}\right]$ of derivations $D$ and $D^{\prime}$ of degrees $\alpha$ and $\alpha^{\prime}$ is a derivation of degree $\alpha+\alpha^{\prime}$. This is done by a simple computation.

Let $E=\oplus_{\alpha} E^{\alpha}$ be a graded Lie algebra. If $a \in E$, we denote by $\operatorname{ad}_{E} a$ (or simply ad $a$ if the meaning is clear) the linear map $x \rightarrow[a, x]$ of $E$ into $E$. The map $a \rightarrow \operatorname{ad}_{E} a$ is called the adjoint map of $E$.

Proposition 5.2. If $a \in E^{\alpha}$, then $\operatorname{ad}_{E} a \in D(E)$. The adjoint map $\operatorname{ad}_{E}: E \rightarrow D(E)$ is a homomorphism of graded Lie algebras. The image $D_{i}(E)$ is an ideal in $D(E)$.

Proof. The first statement follows from (3.2), which is one version of the Jacobi identity. The second statement follows from another version (3.3). If $a \in E^{\alpha}$, and $D \in D^{\beta}(E)$, then an easy computation shows that $\left[D, \operatorname{ad}_{E} a\right]=\operatorname{ad}_{E} D a$. This proves the last statement.

Remarks. 1 . The kernel of the adjoint map is the center of $E$. The graded Lie algebra $D_{i}(E)$ is the graded Lie algebra of inner derivations of $E$. It is isomorphic to the quotient algebra of $E$ modulo its center.

2. The quotient algebra $D(E) / D_{i}(E)$ is called the graded Lie algebra of outer derivations of $E$. It has a natural action on the center of $E$.

6. Complexes defined by derivations of graded algebras. Let $A$ be a graded algebra of type $\Delta$ and let $D$ be a homogeneous derivation of $A$ of degree $\alpha$ with $D \circ D=0$. Then $(A, D)$ is a complex (of type $\Delta$ ) and we can form the cohomology (vector) space in the standard manner. We let $Z(A, D)$ denote the kernel of $D$, and denote the image of $D$ by $B(A, D)$. Then both $Z(A, D)$ and $B(A, D)$ are graded subspaces of $A$, called the spaces of $D$-cocycles and $D$-coboundaries. The quotient space $H(A, D)=Z(A, D) / B(A, D)$ thus has a natural grading; it is the cohomology space of $A$ with respect to $D$.

The fact that $D$ is a derivation implies, by a well-known (and elementary) argument, that $Z(A, D)$ is a subalgebra of $A$ and that $B(A, D)$ is an ideal in $Z(A, D)$. Furthermore, any identities (such as commutativity, associativity, a Jacobi identity, etc.) that hold in $A$ will automatically hold in $H(A, D)$. Thus we obtain:

Proposition 6.1. If $D$ is a derivation of degree $\alpha$ of a graded algebra $A$ such that $D \circ D=0$, then the cohomology space $H(A, D)$ has a graded algebra structure. If $A$ is commutative, anticommutative, associative, or a graded Lie algebra, then so is $H(A, D)$.

Let $E=\oplus_{\alpha} E^{\alpha}$ be a graded Lie algebra and let $a \in E^{\alpha}$ be a homogeneous element of odd degree such that $[a, a]=0$. Since $a \rightarrow \operatorname{ad}_{E} a$ is a homomorphism it follows that $0=\left[\operatorname{ad}_{E} a, \operatorname{ad}_{E} a\right]=2\left(\operatorname{ad}_{E} a \circ \operatorname{ad}_{E} a\right)$, 
hence (recall char $K \neq 2) \operatorname{ad}_{E} a \circ \operatorname{ad}_{E} a=0$. Thus we can form the cohomology space $H\left(E, \operatorname{ad}_{E} a\right)$, which is again a graded Lie algebra. Cohomology spaces of this type will be of particular importance in applications.

7. Semi-direct products. The action of $\mathfrak{D}(E)$ on a graded Lie algebra $E$, together with the graded Lie algebra structures of $E$ and $D(E)$ themselves, can be expressed in one graded Lie algebra structure on the graded vector space $E \oplus D(E)$. More generally, given graded Lie algebras $E$ and $F$, and a homomorphism of $F$ into $D(E)$, a graded Lie algebra structure can be defined on $E \oplus F$ expressing all actions.

Proposition 7.1. If $E$ and $F$ are graded Lie algebras and $f \rightarrow D_{f}$ is a homomorphism of $F$ into $D(E)$, then the graded vector space $E \oplus F$. equipped with the product determined by

$$
[(a, f),(b, g)]=\left([a, b]+D_{f} b-(-1)^{\alpha \beta} D_{0} a,[f, g]\right),
$$

for $a \in E^{\alpha}, b \in E^{\beta}, f \in F^{\alpha}$ and $g \in F^{\beta}$, is a graded Lie algebra. It contains $F$ as a subalgebra, and $E$ as an ideal (i.e. it is a split extension of $F$ by E).

Proof. GLA1, 2 are obvious. The Jacobi identity splits into four pieces containing terms of the following forms: (1) $[[a, b], c]$, (2) $[[f, g], h],(3)[[f, b], c]$ and $(4)[[f, g], c]$. The terms of the form (1) (resp. (2)) drop out because $E$ (resp. $F$ ) is a graded Lie algebra, terms of the form (3) drop out because $D_{f}$ is a derivation on $E$, and terms of the form (4) drop out because $f \rightarrow D_{f}$ is a graded Lie algebra homomorphism. The write-out is straightforward. In case char $K=3$, one must also check $[[(a, f),(a, f)],(a, f)]=0$. It is a simple exercise. The fact that $F$ is a subalgebra and $E$ an ideal is immediate from the product formula.

The graded Lie algebra constructed above is called the semi-direct product of $E$ and $F$ with respect to the representation $f \rightarrow D_{f}$.

Conversely, every split extension of $F$ by $E$ is isomorphic to a suitable semi-direct product of $E$ and $F$.

We return now to the situation considered in $\S 6$. Let $E$ be a graded Lie algebra and let $D$ be a derivation of $E$ of odd degree $\alpha$ such that $D \circ D=0$. Then $K D$ (the subspace of $D(E)$ spanned by $D$ ) is a graded subalgebra of $D(E)$, since $[D, D]=D \circ D-(-1)^{\alpha \alpha} D \circ D$ $=2 D \circ D=0$. We can thus take the semi-direct product $E \oplus K D$ (with respect to the inclusion homomorphism $K D \rightarrow D(E)$ ). The derivation $D$ can be considered as the restriction to $E$ of an inner derivation of $E \oplus K D$. We note that if $D=0$, then $E \oplus K D=E$. 
8. The general linear group of a graded vector space. Let $V=\oplus_{\alpha \in \Delta} V^{\alpha}$ be a graded vector space. We define $\operatorname{GL}(V)$, the general linear group of $V$, to be the group of all homogeneous automorphisms of degree 0 of $V$. GL $(V)$ is canonically isomorphic to the direct product of the family $\left\{\mathrm{GL}\left(V^{\alpha}\right)\right\}_{\alpha \in \Delta}$ of groups. If $G$ is any group, then a homomorphism $\rho: G \rightarrow \mathrm{GL}(V)$ is uniquely determined by a family $\left\{\rho^{\alpha}\right\}_{\alpha \in \Delta}$, where, for each index $\alpha, \rho^{\alpha}$ is a homomorphism $G \rightarrow \operatorname{GL}\left(V^{\alpha}\right)$. We write $\rho=\left\{\rho^{\alpha}\right\}_{\alpha \in \Delta \text {. }}$

Consider now the case in which each direct summand $V^{\alpha}$ of $V$ is finite-dimensional. Let $G$ be an algebraic group with Lie algebra $\mathfrak{g}$. A homomorphism $\rho=\left\{\rho^{\alpha}\right\}_{\alpha \in \Delta}$ of $G$ into $\mathrm{GL}(V)$ is a rational representation of $G$ on $V$ if, for every index $\alpha, \rho^{\alpha}$ is a rational representation of $G$ on $V^{\alpha}$. For each $\alpha$, the differential $d \rho^{\alpha}$ is a homomorphism of $\mathfrak{g}$ into $\mathfrak{g l}\left(V^{\alpha}\right)$. The family $\left\{d \rho^{\alpha}\right\}_{\alpha \in \Delta}$ of homomorphisms determines a unique homomorphism $d \rho$ of $\mathfrak{g}$ into the Lie algebra $\mathfrak{g l}^{0}(V)$ of all homogeneous endomorphisms of degree 0 of $V$; we call $d \rho$ the differential of the rational representation $\rho$.

Let the base field of $V$ be either $R$ or $C$ and let $G$ be a (real or complex) Lie group with Lie algebra g. A homomorphism $\rho=\left\{\rho^{\alpha}\right\}_{\alpha \in \Delta}$ of $G$ into $\mathrm{GL}(V)$ is a representation of $G$ on $V$ if, for every $\alpha, \rho^{\alpha}: G$ $\rightarrow \mathrm{GL}\left(V^{\alpha}\right)$ is a homomorphism of (real or complex) Lie groups. As above we define the differential $d \rho$ to be the homomorphism of $\mathfrak{g}$ into $\mathfrak{g l}^{0}(V)$ determined by the family $\left\{d \rho^{\alpha}\right\}_{\in \alpha \Delta}$ of homorphisms.

\section{Analytic graded Lie algebras.}

Definition 9.1. An analytic graded Lie algebra is a triple $(E, G, \rho)$, where $E=\bigoplus_{\alpha} E^{\alpha}$ is a graded Lie algebra over either $R$ or $C, G$ is a (real or complex) Lie group with Lie algebra $E^{0}$, and $\rho$ is a continuous homomorphism of $G$ into $\mathrm{GL}(E)$, subject to the following conditions:

(i) Each direct summand $E^{\alpha}$ of $E$ is finite-dimensional;

(ii) $\rho$ is a representation of $G$ on $E$;

(iii) The differential $d \rho$ of $\rho$ is just the homomorphism $\operatorname{ad}_{E}$ of $E^{0}$ into $\mathrm{gl}^{0}(V)$;

(iv) Each $\rho(g)$, for $g \in G$, is an automorphism of $E$.

We note that condition (iv) is a consequence of the other conditions if $G$ is connected. This follows from the fact that each $\operatorname{ad}_{E} a$ is a derivation (of degree 0 ) of $E$. The proof is similar to that of $[1$, p. 136]. (One must be careful, however, since $E$ is, in general, infinite-dimensional.)

$G$ is called the structure group of $(E, G, \rho) ; \rho(G)$ is the group of inner automorphisms of $(E, G, \rho)$.

Theorem 9.2. Let $E$ be a graded Lie algebra over $\boldsymbol{R}$ or $\boldsymbol{C}$ satisfying condition (i). Then there exists a connected and simply connected Lie 
group $G$ and a representation $\rho$ of $G$ on $E$ such that $(E, G, \rho)$ is an analytic graded Lie algebra. If $\left(E, G^{\prime}, \rho^{\prime}\right)$ is another analytic graded Lie algebra with underlying graded Lie algebra $E$, then there exists a unique homomorphism $h: G \rightarrow G^{\prime}$ onto the identity component of $G^{\prime}$ with discrete kernel such that $\rho=\rho^{\prime} \circ h$.

The proof of Theorem 9.2 follows easily from standard theorems in the theory of Lie groups. We omit details.

Let $E$ be as above. It follows that the identity component of the group of inner automorphisms of $(E, G, \rho)$ is uniquely determined by $E$.

10. Algebraic graded Lie algebras. The algebraic analogue of Definition 9.1 is the following.

DeFINITION 10.1. An algebraic graded Lie algebra over a field $K$ is a triple $(E, G, \rho)$, consisting of a graded Lie algebra $E=\bigoplus_{\alpha} E^{\alpha}$ over $K$, an algebraic group $G$ over $K$ with Lie algebra $E^{0}$, and a homomorphism $\rho$ of $G$ into $G L(E)$, subject to the following conditions:

(i) Each direct summand $E^{\alpha}$ of $E$ is finite-dimensional;

(ii) $\rho$ is a rational representation of $G$ on $E$;

(iii) The differential $d \rho$ is the map $\operatorname{ad}_{E}$ of $E^{0}$ into $\mathrm{gl}^{0}(E)$;

(iv) Each $\rho(g)$, for $g \in G$, is an automorphism of $E$.

In the analytic case if $E$ is a graded Lie algebra satisfying (i), then by Theorem 9.1 there exists an analytic graded Lie algebra with underlying graded algebra $E$. Furthermore, this analytic graded Lie algebra is to a large extent unique. If $E$ is a graded Lie algebra satisfying condition (i) over a field $K$, there is in general no algebraic graded Lie algebra with underlying graded Lie algebra $E$. Furthermore, if such algebraic graded Lie algebras do exist there is no analogue of the uniqueness part of Theorem 9.2. In the analytic case for connected $G$ we saw that condition (iv) was a consequence of the other hypotheses. In the algebraic case this is no longer true.

11. Admissible derivations. Let $(E, G, \rho)$ be an analytic (resp. algebraic) graded Lie algebra.

DEFINITION 11.1. An admissible derivation of degree $\alpha$ of $(E, G, \rho)$ is a pair $\left(D, \rho^{\prime}\right)$ consisting of a derivation $D$ of $E$ of odd degree $\alpha$ satisfying $[D, D]=0$ and a representation (resp. rational representation) $\rho^{\prime}$ of $G$ on the semi-direct product $E \oplus K D=E^{\prime}$ such that the following conditions are satisfied for each $g \in G$ :

(i) Each $\rho^{\prime}(g)$ is an automorphism of the graded Lie algebra $E^{\prime}$;

(ii) The restriction of $\rho^{\prime}(g)$ to $E$ is identical with $\rho(g)$;

(iii) The endomorphism of the quotient space $E^{\prime} / E$ induced by $\rho^{\prime}(g)$ is the identity; 
(iv) The differential of $\rho^{\prime}$ is the homomorphism $\operatorname{ad}_{E^{\prime}}$ of $E^{0}$ into $\mathfrak{g l}\left(E^{\prime}\right)$.

The above conditions imply that $\left(E^{\prime}, G, p^{\prime}\right)$ is an analytic (resp. algebraic) graded Lie algebra. We note that the zero derivation is an admissible derivation of arbitrary (odd) degree (set $\rho^{\prime}=\rho$ ).

Let $(E, G, \rho)$ be an analytic graded Lie algebra such that $G$ is simply connected (and connected). Corresponding to every derivation $D$ of odd degree such that $D \circ D=0$ there exists a unique representation $\rho^{\prime}$ of $G$ on $E^{\prime}$ such that $\left(D, \rho^{\prime}\right)$ is an admissible derivation. For, since $G$ is simply connected, there exists a unique representation $\rho^{\prime}$ of $G$ on $E^{\prime}$ satisfying (iv). Condition (ii) follows immediately and (i) follows from the fact that, for each $a \in E^{0}, \operatorname{ad}_{E^{\prime}} a$ is a derivation of $E^{\prime}$. Finally, (iii) is a consequence of the fact that $\operatorname{ad}_{E^{\prime}} a$ induces the zero map on $E^{\prime} / E$.

We note that the analogous statement for algebraic graded Lie algebras is not true.

\section{\$2. Graded Lie algebras OVER FIELdS OF Characteristic 2}

In this part we indicate the modifications necessary in our definitions of $\$ 1$ if the base field is of characteristic 2. Cross-references to $\$ 1$ have been simplified by following a similar numbering of sections.

2 '. Commutative and anticommutative graded algebras. Oddness and evenness of elements of the grading group $\Delta$ is defined as in $\$ 1$. A graded algebra is called weakly commutative (resp. weakly anticommutative) if $a b=b a$ for all $a, b \in A$, and $a a=0$ for all $a \in A^{\alpha}$ and $\alpha$ odd (resp. $\alpha$ even).

The concept of strong commutativity (also called commutativity) is defined by requiring that there is given a quadratic operation $Q[a]$ for $a$ of even degree, which generalizes the expression $\frac{1}{2} a a$ when the base field has characteristic $\neq 2$. Similarly, for strong anticommutativity (also called anticommutativity) $Q[a]$ is defined for $a$ of odd degree.

A strongly commutative (resp. strongly anticommutative) graded algebra is a graded algebra $A$ in which, besides the bilinear product $A \times A \rightarrow A$ there is defined a map $Q: A^{\prime} \rightarrow A$, where $A^{\prime}$ is the direct sum of the $A^{\alpha}$ with even $\alpha$ (resp. odd $\alpha$ ), satisfying:

SA1. $a b=b a$ for all $a, b \in A$;

SA2. $a a=0$ for all $a \in A^{\alpha}$ and $\alpha$ odd (resp. $\alpha$ even);

SA3. $Q\left[A^{\alpha}\right] \subset A^{2 \alpha}$ for $\alpha$ even (resp. $\alpha$ odd);

SA4. $Q[k a]=k^{2} Q[a]$ for $k \in K, a \in A^{\prime}$;

SA5. $Q[a+b]=Q[a]+Q[b]+a b$ for $a, b \in A^{\prime}$.

Condition SA5 with $a=b$ gives $a a=Q[2 a]+2 Q[a]=0$ for $a \in A^{\prime}$. 
In view of SA2 this means that all squares vanish.

Associativity for weakly commutative (resp. weakly and strongly anticommutative) graded algebras is as before: $a(b c)=(a b) c$; for the (strong) commutative case the additional condition $Q[a b]=0$ for all products $a b$ in $A^{\prime}$ is imposed.

$3^{\prime}$. Graded Lie algebras. The quadratic map of a (strong) graded Lie algebra has to satisfy an extra "Jacobi" identity.

A weak graded Lie algebra over a field $K$ (char $K=2$ ) is a weak anticommutative graded algebra whose product satisfies the Jacobi identity GLA3 of $\$ 3$. A (strong) graded Lie algebra over $K$ is a strongly anticommutative graded algebra whose product satisfies GLA3 of $\S 3$ add whose quadratic map satisfies

GLA4. $[a,[a, b]]=[Q[a], b]$ when $a$ is of odd degree.

REMARK. The distinction is made between weak subalgebras and (strong) subalgebras, ditto for homomorphisms, ideals, etc., depending on whether the quadratic operation is preserved. The quotient of a graded Lie algebra modulo a weak (resp. strong) ideal is a weak (resp. strong) graded Lie algebra.

Proposition $3^{\prime}$.1. If $E$ is a weak graded Lie algebra, then the squares $[a, a]$ of all belong to the center.

Proof. When $a$ is of even degree, then $[a, a]=0$. When $a$ is of odd degree, then we apply the Jacobi identity (3.3) for arbitrary $b$ :

$$
[a,[a, b]]+[a,[a, b]]=[[a, a], b] .
$$

The left side equals $2[a,[a, b]]$, which vanishes.

$4^{\prime}$. Graded Lie algebras of commutators. Let $V$ be a graded vector space.

Proposition $4^{\prime} .1$. The graded algebra $\mathfrak{g l}(V)$ whose direct summands $\mathfrak{g l}^{\alpha}(V)$ are the spaces $\operatorname{Hom}^{\alpha}(V, V)$, and in which the product and quadratic operation are defined by

$$
\begin{aligned}
{[f, g] } & =f \circ g+g \circ f ; \\
Q[f] & =f \circ f \text { for } f \text { of odd degree; }
\end{aligned}
$$

is a (strong) graded Lie algebra.

Proof. The verification of the anticommutativity property of the product, and the conditions on $Q$ : additivity, quadratic behavior under scalar multiplication and GLA4, are elementary.

Propositions 4.1 and 4.2 remain true, with $Q[a]$ in the graded Lie algebra defined as $a a$. 
$5^{\prime}$. Graded Lie algebras and derivations. A weak derivation on a graded algebra $A$ is a map $D: A \rightarrow A$ as in $\$ 5$. A (strong) derivation of a commutative (resp. anticommutative) graded algebra $A$ is a map $D: A \rightarrow A$ as in $\$ 5$ satisfying the condition

$$
D Q[a]=(D a) a \text { for } a \in A^{\prime} .
$$

Proposition 5'.1. The weak derivations of a graded algebra $A$ form a (strong) subalgebra of $\mathfrak{g l}(A)$.

Proof. The additional fact to be shown is that $Q[D]=D \circ D$ is a derivation when $D$ is of odd degree. It follows immediately by direct computation.

Proposition 5'.2. The strong derivations of a strong commutative (resp. anticommutative) graded algebra $A$ form a strong subalgebra of $\mathfrak{g l}(A)$.

Proof. It has to be shown that $\left[D, D^{\prime}\right] Q[a]$, which by definition is $D D^{\prime} Q[a]+D^{\prime} D Q[a]$, equals $\left(\left[D, D^{\prime}\right] a\right) a$; furthermore, it must be shown that $D D Q[a]=(D D a) a$; i.e. $Q[D] Q[a]=(Q[D] a) a$. Both verifications are routine; the first uses $(D a)\left(D^{\prime} a\right)=\left(D^{\prime} a\right)(D a)$ and the second uses the vanishing of all squares, so $(D a)(D a)=0$.

Proposition 5'.3. If a weak graded Lie algebra $E$ has no center, then there exists a minimal strong graded Lie algebra $\bar{E}$ which contains $E$ as a weak subalgebra.

Proof. The adjoint map $\operatorname{ad}_{E}: E \rightarrow D(E)$ is an injection; $D(E)$ is strong. Let $\bar{E}$ be the subspace of $\mathscr{D}(E)$ spanned by $\operatorname{ad}_{E}(E)$ and the elements $Q\left[\operatorname{ad}_{E} a\right]$ for all $a \in E$ of odd degree. Then $\bar{E}$ is a subalgebra. Indeed, [ad $E$, ad $E] \subset$ ad $E \subset \bar{E}$, and

$$
[Q[\operatorname{ad} a], \operatorname{ad} E]=[\operatorname{ad} a,[\operatorname{ad} a, \operatorname{ad} E]] \subset \operatorname{ad} E \subset \bar{E} ;
$$

also

$$
\begin{aligned}
{[Q[\operatorname{ad} a], Q[\operatorname{ad} b]] } & =[\operatorname{ad} a,[\operatorname{ad} a, Q[\operatorname{ad} b]]] \\
& =[\operatorname{ad} a,[\operatorname{ad} b,[\operatorname{ad} b, \operatorname{ad} a]]] \in \operatorname{ad} E \subset \bar{E} .
\end{aligned}
$$

$\bar{E}$ is strong, since $\bar{E}^{\alpha}=$ ad $E^{\alpha}$ for $\alpha$ odd.

Let $F_{1}$ be a strong graded Lie algebra, and contain $E$ as a weak subalgebra. Then the set $F_{2}$ of $f \in F_{1}$ such that $[f, E] \subset E$ is the normalizer of $E$, and is easily seen to be a strong graded Lie algebra. Since $F_{2}$ acts on $E$ as derivations, there is a natural homomorphism $\phi: F_{2}$ $\rightarrow D(E)$ which sends $E$ onto ad $E$. It is a strong homomorphism, so the image covers $\bar{E}$. It follows that $\bar{E}$ is the homomorphic image of a subalgebra (namely, $\phi^{-1}(\bar{E})$ ) of $F_{1}$. 
$6^{\prime}$. Complexes defined by derivations of graded algebras. When $A$ is a strong graded commutative (resp. anticommutative) algebra, and $D$ a strong derivation satisfying $D \circ D=0$, of homogeneous degree $\alpha$, then $Q[a]$ is a $D$-cocycle if $a$ is a $D$-cocycle and if the degree of $a$ is even (resp. odd). The ideal $B(A, D)$ in $Z(A, D)$ is, in general, not closed under the quadratic map, however. Thus, cohomology spaces of strong commutative (resp. anticommutative) graded algebras are in general only weak algebras of the same type. The quotient $Z(A, D) / \bar{B}(A, D)$ is a strong algebra, however, if $\bar{B}(A, D)$ is the subspace (ideal) in $Z(A, D)$ spanned by $B(A, D)$ and the elements $Q[a]$ for all $a \in B(A, D)$ of even (resp. odd) degrees.

The construction of cohomology spaces $H\left(E, \operatorname{ad}_{E} a\right)$ analogous to that in $\S 6$ is possible if $E$ is a strong graded Lie algebra, if $a$ is homogeneous of odd degree and if $Q[a]=0$.

$10^{\prime}$. Algebraic graded Lie algebras. Weak algebraic graded Lie algebras are defined as in $\$ 10$; strong algebraic graded Lie algebras admit, in addition, the usual quadratic map, and the representation $\rho$ must satisfy the additional condition

(v) $\rho(g) Q[a]=Q[\rho(g) a]$ for all $g \in G$.

\section{§3. The Deformation PRoblem}

12. The deformation equation. Let $E=\oplus_{\alpha} E^{\alpha}$ be a graded Lie algebra over a field $K$ of characteristic $\neq 2$ and let $D$ be a derivation of $E$ of odd degree $\alpha$ such that $D \circ D=0$. The equation

$$
D a+\frac{1}{2}[a, a]=0, \quad a \in E^{\alpha}
$$

is called the deformation equation. We denote by $M_{0}$ the set of all solutions of (12.1):

$$
M_{0}=\left\{a \in E^{\alpha} \mid D a+\frac{1}{2}[a, a]=0\right\} .
$$

Let $E^{\prime}$ be the semi-direct product $E \oplus K D$ and let $L$ be the linear variety in $E^{\prime}$ parallel to $E^{\alpha}$ and passing through $D$, i.e. $L=D+E^{\alpha}$. Let

$$
M=\{x \in L \mid[x, x]=0\} .
$$

If $x=D+a$ is an element of $L$, then

$$
[x, x]=[D+a, D+a]=2 D a+[a, a] ;
$$

thus $x \in M$ if and only if $a \in M_{0}$. Hence the affine bijection $\eta: a \rightarrow D+a$ of $E^{\alpha}$ onto $L$ maps $M_{0}$ onto $M$. Instead of studying the set $M_{0}$ of all solutions of the deformation equation, it is sometimes more convenient to consider instead the set $M$.

If $x=D+a$ is an element of $M$, we denote by $D_{x}$ the derivation 
$D+\operatorname{ad}_{E} a$ of $E$ i.e. $D_{x}$ is the restriction of $\operatorname{ad}_{E} x$ to $E$. It follows from the definition of $M$ that $D_{x} \circ D_{x}=0$; thus we can associate to each $x \in M$ the cochain complex $\left(E, D_{x}\right)$ and the cohomology space $H\left(E, D_{x}\right)$.

The simplest case of the deformation equation, but a very important one for applications, occurs when $D=0$. In this case $M=M_{0}$, and $D_{x}$ is the inner derivation $\operatorname{ad}_{E} x$.

In the rest of this paper we shall study the set $M_{0}$ of solutions of the deformation equation, or equivalently the set $M$ described above, when $E$ (really: $(E, G, \rho)$ ) is an analytic or algebraic graded Lie algebra and $D$ (really: $\left(D, \rho^{\prime}\right)$ ) is an admissible derivation. In the algebraic case the field $K$ is assumed to be algebraically closed. In either case $M$ is an algebraic set in $L$. It follows from the definition of an admissible derivation that both $L$ and $M$ are stable under the action of $G$ on $E^{\prime}$ determined by the representation $\rho^{\prime}$. Hereafter we consider both $L$ and $M$ as transformation spaces for $G$ and suppress mention of $\rho^{\prime}$; if $g \in G$ and $x \in L$, we denote $\rho^{\prime}(g) x$ simply by $g \cdot x$.

If we wish to work with the set $M_{0}$ of solutions of (12.1), we proceed as follows: By means of the affine bijection $\eta$ of $E^{\alpha}$ onto $L$, we assign to each $g \in G$ an affine transformation $T_{g}$ of $E^{\alpha}$. Precisely, $T_{g}$ is the affine transformation $a \rightarrow \eta^{-1}(g \cdot \eta(a))$ of $E^{\alpha}$. Then the map $g \rightarrow T_{g}$ is an affine representation of $G$ on $E^{\alpha}$ and $M_{0}$ is stable under the action of $G$ on $E^{\alpha}$ determined by this representation.

For a local study of $M$ we take a point $m=D+a$ on $M$, and set $x=m+u ;$ then

$$
[x, x]=[m+u, m+u]=2[m, u]+[u, u]=2 D_{m} u+[u, u],
$$

so $x \in M$ if and only if $u$ satisfies

$$
D_{m} u+\frac{1}{2}[u, u]=0
$$

the "small" solutions $u$ of this deformation equation relative to $m$ give a neighborhood of $m$ on $M$.

When the base field $K$ has characteristic 2, and when $E$ is a strong graded Lie algebra, the deformation equation reads

$$
D a+Q[a]=0, \quad a \in E^{\alpha}
$$

in this case $M$ is the set $\{x \in L \mid Q[x]=0\}$.

Hereafter we shall assume that char $K \neq 2$. The results which we obtain can be translated to the case in which char $K=2$ by the standard changes elaborated in $\$ 2$. They will not be mentioned each time.

13. Problems leading to the deformation equation. To each vector space $V$ over a field $K$ can be associated a graded Lie algebra 
$E=E_{1 \text { in }}(V)$ of type $Z$ whose direct summand $E^{n}$ consist of the $(n+1)$ multilinear maps of $V$ into $V$. The product in $E$ is such that a bilinear map $\mu \in E^{1}$ of $V$ into $V$ defines an associative multiplication on $V$ if and only if $[\mu, \mu]=0$. (If char $K=2$, the condition reads $Q[\mu]=0$.) Thus the set $M=\left\{\mu \in E^{1} \mid[\mu, \mu]=0\right\}$ is precisely the set of all associative multiplications on $V$. If $\mu \in M$ and if $A=(V, \mu)$ is the corresponding associative algebra, then the cohomology space $H^{n}\left(E, D_{\mu}\right)$ is identical with the cohomology space $H^{n+1}(A, A)$ defined by Hochschild [11]. If $V$ is finite-dimensional, then $E_{1 \text { in }}(V)$ admits a natural structure of algebraic graded Lie algebra with structure group $\mathrm{GL}(V)$. Furthermore, two elements $\mu_{1}$ and $\mu_{2}$ of $M$ lie on the same orbit under $\mathrm{GL}(V)$ if and only if the corresponding associative algebras are isomorphic. The graded Lie algebra structure on $E_{\text {lin }}(V)$ was established by Gerstenhaber [8].

A similar situation exists for the case of Lie algebra multiplications on $V$. In this case there exists a graded Lie algebra $E=E_{\text {alt }}(V)$ whose direct summand $E^{n}$ consists of all alternating $(n+1)$-multilinear maps of $V$ into $V$. An element $\mu \in E^{1}$ satisfies the Jacobi identity if and only if $[\mu, \mu]=0$. If $\mu$ satisfies the above equation and if $A=(V, \mu)$ is the corresponding Lie algebra, then the cohomology space $H^{n}\left(E, D_{\mu}\right)$ is identical with the cohomology space $H^{n+1}(A, A)$ as defined by Chevalley-Eilenberg [5]. Again, if $V$ is finite-dimensional, then $E_{\text {alt }}(V)$ admits the structure of an algebraic graded Lie algebra with structure group GL(V); two Lie algebra multiplications lie on the same orbit under GL( $V)$ if and only if the corresponding Lie algebras are isomorphic.

A similar situation arises for multiplications on $V$ which are commutative and associative. In this case the cohomology spaces which arise are those recently defined by Harrison [10].

The deformation equation also arises in the study of homomorphisms of algebras. Let $A$ and $B$ be algebras (either associative or Lie). Then there exists a graded Lie algebra $E$ such that $E^{1}$ is the set of all linear maps of $A$ into $B$. Furthermore, there exists an (outer) derivation $D$ of $E$ such that an element $\phi$ of $E^{1}$ is a homomorphism of $A$ into $B$ if and only if $D \phi+\frac{1}{2}[\phi, \phi]=0$. Let $\phi \in E^{1}$ be a homomorphism and let $x=D+\phi$. Then $H^{n}\left(E, D_{x}\right)$ is identical with the cohomology space $H^{n}(A, B)$, where $B$ is considered as an $A$ module via $\phi$. Let $A$ and $B$ be finite dimensional, and let the base field be either $\boldsymbol{R}$ or $\boldsymbol{C}$, then $E$ admits a natural structure of analytic graded Lie algebra whose structure group $G$ is the group of inner automorphisms of $B$. Furthermore, two homomorphisms lie on the same orbit under $G$ if and only if they differ by an inner automor- 
phism of $B$. However, if $A$ and $B$ are Lie algebras and the base field is arbitrary, $E$ will not, in general, admit a structure of algebraic graded Lie algebra. A sufficient condition that $E$ should admit such a structure is that $B$ be an algebraic Lie algebra (i.e. the Lie algebra of an algebraic group).

14. The deformation language. In this section $E$ is an analytic or algebraic graded Lie algebra over a field $K$ (algebraically closed in the algebraic case) and $D$ is an admissible derivation of $E$. $L$ and $M$ are as defined in $\$ 12$; the structure group $G$ acts on $L$ and $M$ via the representation $\rho^{\prime}$. In the analytic case $L$ is given the usual topology of a finite dimensional real or complex affine space and $M$ is given the induced topology. In the algebraic case $L$ and $M$ are given the Zariski topology. The elements $x$ and $y$ of $M$ are said to be equivalent if they lie on the same orbit under $G$.

In the examples discussed in $\$ 13$ each element of $M$ represented a certain type of algebra structure; two elements of $M$ were equivalent if and only if the corresponding algebra structures were isomorphic. If $m \in M$ and $N$ is a neighborhood of $m$ on $M$, then each point $x$ of $N$ can intuitively be considered as a deformation of the structure represented by $m$; the deformation is trivial if $x$ is equivalent to $m$, that is if the corresponding structures are isomorphic.

We shall consistently use the intuitive language suggested by the above discussion. Thus, for example, an element $m \in M$ is rigid if it admits (locally) no nontrivial deformations, i.e. if the orbit $G(m)$ is an open subset of $M$. By a family of deformations of $m \in M$ we shall mean a connected subset $F$ of $M$ which contains $m$. The family $F$ of deformations of $m$ is locally complete at $m$ if the orbit $G(F)$ is a neighborhood of $m$ on $M$.

In $\$ 4$ we shall construct a locally complete family of deformations of $m$ parametrized (at least in the analytic case) by an analytic subset of $H^{1}\left(E, D_{m}\right)$.

In our results concerning the deformation equation, only the direct summands $E^{n \alpha}(0 \leqq n \leqq 3)$ are relevant. Thus there is no loss of generality in using $n$ as a grading index and in considering $D$ as a derivation of degree 1. The case when $n \alpha=0$ for some $n$ creates little difficulty, since a formal change (replacing $E^{n \alpha}$ by $E^{n \alpha} \times\{n\}$ ) can make $n$ a grading index.

\section{\$4. Deformation THEOREMS For ANALYTIC GRADED LIE ALGEBRAS}

15. Notation. The following notation will be used throughout $\$ 4$. 
$E=\oplus_{n \in z} E^{n}$ is an analytic graded Lie algebra with structure group $G$ and $D$ is an admissible derivation of $E$ of degree 1 .

$E^{\prime}$ denotes the semi-direct product $E \oplus K D$ (here $K$ is either $R$ or $C): L$ is the linear variety in $E^{\prime}$ parallel to $E^{1}$ and passing through $D$, and $M=\{x \in L \mid[x, x]=0\}$.

If $x \in L$ and $g \in G$, we denote $\rho^{\prime}(g) x$ simply by $g \cdot x$. The exponental map of Lie algebra into Lie group is denoted exp: $E^{n} \rightarrow G$.

For each $m \in M$ we denote by $D_{m}$ the restriction of $\operatorname{ad}_{E^{\prime}}, m$ to $E$; $Z(m)=\oplus_{n} Z^{n}(m)$ denotes the kernel of $D_{m}$ and $B(m)=\oplus_{n} B^{n}(m)$ denotes the image of $D_{m}$. Furthermore, let $H(m)$ be a graded supplementary subspace to $B(m)$ in $Z(m)$, and let $C(m)$ be a graded supplementary subspace to $Z(m)$ in $E$. Set $H^{n}(m)=E^{n} \cap H(m)$ and $C^{n}(m)$ $=E^{n} \cap C(m)$. Note that $H^{n}(m)$ is canonically isomorphic to $H^{n}\left(E, D_{m}\right)$ and that $D_{m}$ induces an isomorphism of $C^{n}(m)$ onto $B^{n+1}(m)$. The direct sum decomposition

$$
E=B(m) \oplus H(m) \oplus C(m)
$$

is called a Hodge decomposition of $E$ with respect to $m$. The symbols $\pi_{B(m)}, \pi_{H(m)}, \pi_{C(m)}$ denote the projection operators corresponding to this Hodge decomposition. When the context is clear, we shall often omit reference to $m$ and write $B^{n}, H^{n}, C^{n}, \pi_{H}$, etc.

All finite-dimensional affine spaces (over $R$ and $C$ ) are given their usual topology; subsets are given the induced topology. If $X$ is a topological space and $x \in X$, the symbols $N(x, X), N_{1}(x, X)$, etc. will denote neighborhoods of $x$ in $X$; if $V$ is a vector space, $N(V)$, etc. will denote a neighborhood of the origin 0 of $V$.

If $f: V \rightarrow W$ is a differentiable map of vector spaces (resp. affine spaces), we denote by $d f_{x}$ the differential of $f$ at the point $x$; i.e. the linear approximation to $f$ at $x$. In the affine case this is a map of the tangent spaces.

We shall make frequent use of the inverse function theorem and the implicit function theorem. We use the formulations given by Dieudonne [6] and Lang [15, Corollary 2, p. 15], with the obvious modifications for maps of affine spaces.

16. The Rigidity Theorem. Let $E=B \oplus H \oplus C$ be a Hodge decomposition with respect to $m \in M$.

Lemma 16.1. Let $m \in M$, then there exist $N(m, L), N\left(C^{0}\right), N\left(H^{1}\right)$ and $N\left(C^{1}\right)$ such that the analytic mapping $F: C^{0} \times H^{1} \times C^{1} \rightarrow L$ given by

$$
F(a, h, u)=\exp (a) \cdot(m+h+u)
$$

maps $N\left(C^{0}\right) \times N\left(H^{1}\right) \times N\left(C^{1}\right)$ diffeomorphically onto $N(m, L)$. 
Proof. By the inverse function theorem it is sufficient to show that the differential $d F_{(0,0,0)}$ of $F$ at the origin is an isomorphism of $C^{0} \times H^{1} \times C^{1}$ onto $E^{1}$. An easy computation shows

$$
d F_{(0,0,0)}(a, h, u)=[a, m]+h+u=-D_{m} a+h+u .
$$

Since $D_{m}$ maps $C^{0}$ isomorphically onto $B^{1}$, and since $E^{1}=B^{1} \oplus H^{1} \oplus C^{1}$, it follows that $d F_{(0,0,0)}$ is an isomorphism.

Theorem 16.2 (Rigidity Theorem). Let $m \in M$ be such that $H^{1}\left(E, D_{m}\right)=0$. Then $m$ is a rigid element of $M$; in fact there exist $N(m, M)$ and $N\left(C^{0}\right)$ such that every element of $N(m, M)$ is of the form $\exp (a) \cdot m$, where $a \in N\left(C^{0}\right)$ and $C^{0}$ is a supplementary space to $Z^{0}(m)$ in $E^{0}$.

Proof. Choose $N_{1}(m, L), N\left(C^{0}\right), N_{1}\left(C^{1}\right)$ so that the statement of the lemma holds. Then every $x \in N_{1}(m, M)$ is of the form $x=\exp (a)$ $\cdot(m+u)$, where $a \in N\left(C^{0}\right)$ and $u \in N_{1}\left(C^{1}\right)$. Furthermore, $x \in M$ if and only if $m+u \in M$. We note that $m=m+0$ belongs to $M$.

Let $P$ denote the polynomial mapping $u \rightarrow[m+u, m+u]$ of $C^{1}$ into $E^{2}$; we note that $m+u \in M$ if and only if $P(u)=0$. The differential $d P_{0}$ is just $D_{m}$; hence $d P_{0}$ is an injection. It follows from the inverse function that there exists an $N_{2}\left(C^{1}\right)$ such that the restriction of $P$ to $N_{2}\left(C^{1}\right)$ is an injection. Thus, if $u \in N_{2}\left(C^{1}\right)$, then $m+u \in M$ if and only if $u=0$. Now take $N\left(C^{1}\right)=N_{1}\left(C^{1}\right) \cap N_{2}\left(C^{1}\right)$ and $N(m, L)=\exp \left(N\left(C^{0}\right)\right)$ $\cdot N\left(C^{1}\right)$, and set $N(m, M)=N(m, L) \cap M$; then every $x \in N(m, M)$ is of the form $\exp (a) \cdot m$ with $a \in N\left(C^{0}\right)$.

17. Linear Lie groups acting on algebraic sets. The proposition proved in this section allows us to strengthen the conclusion of the Rigidity Theorem.

First we recall some facts about algebraic sets in complex $n$-dimensional space $C^{n}$. Let $S$ be an irreducible algebraic set in $C^{n}$ and let $S_{0}$ be the set of simple points of $S$. (For the definition of simple point, see §21.) $S_{0}$ is a Zariski-open subset of $S$. If $x \in S_{0}$, then there is a neighborhood $N\left(x, \mathbf{C}^{n}\right)$ such that $S \cap N\left(x, \mathbf{C}^{n}\right)$ is a complex submanifold of $N\left(x, C^{n}\right)$. If $x \in S_{0}$, then the tangent space to $S_{0}$ (considered as a complex submanifold of $C^{n}$ ) at $x$ is identical with the (Zariski) tangent space of $S$ (considered as an algebraic subvariety of $\boldsymbol{C}^{n}$ ) at $x$; in particular the dimension of $S_{0}$ as a complex manifold is equal to the dimension of $S$ as an algebraic variety. If $U$ is a Zariski-open subset of $S$, then $U$ is connected (in the topology induced on $U$ by the usual topology of $\mathbf{C}^{n}$ ). For a proof of these statements we refer the reader to [18, pp. 163-169].

If $S$ is an irreducible algebraic set in $R^{n}$, then all the statements in 
the above paragraph hold except the last one. In this case one can only say that $U$ has a finite number of components. For details, see [18].

Proposition 17.1. Let $S$ be an algebraic set in $\mathbf{C}^{n}$ (resp. $\boldsymbol{R}^{n}$ ) and let $G$ be a Lie subgroup of $\mathrm{GL}\left(\boldsymbol{C}^{n}\right)$ (resp. $\mathrm{GL}\left(\boldsymbol{R}^{n}\right)$ ) such that $S$ is stable under the action of $G$. Suppose there is a point $x \in S$ such that the orbit $G(x)$ is an open subset of $S$. Then $G(x)$ is a Zariski-open subset of $S$ (resp. is one component of a Zariski-open subset of $S$ ).

Proof. We may assume that $G$ is connected. We first give the proof for $S \subset C^{n}$. It is easily seen that each irreducible component of $S$ is stable under the action of $G$. Thus we may assume that $S$ is irreducible. Let $k$ be the dimension of $S$ as an algebraic variety and let $\mathfrak{g}$ denote the Lie algebra of $G$. For each $x \in C^{n}$ the orbit $G(x)$ is a complex submanifold of $\boldsymbol{C}^{n}$; the (differential-geometric) tangent space to $G(x)$ at $x$ is just $\mathfrak{g}(x)=\{T(x) \mid T \in \mathfrak{g}\}$. If $x \in S$, then it follows easily that $\operatorname{dim} \mathfrak{g}(x) \leqq k$. Let $U=\{x \in S \mid \operatorname{dim} \mathfrak{g}(x)=k\}$. An elementary argument shows that $U$ is a Zariski-open subset of $S$; furthermore, $U$ is nonempty, since it contains the given point $x$. It is easy to see that $U$ is included in the set $S_{0}$ of simple points of $S$. If $x \in U$, then the orbit $G(x)$ is an open subset of $U$; thus the orbits of $G$ partition $U$ into disjoint open sets. Since $U$ is connected, there is precisely one orbit of $G$ on $U$.

If $S \subset R^{n}$, then the above argument is valid except for the last sentence. In this case $U$ has only a finite number of components and the conclusion of the theorem follows easily.

We note that in either case $\left(S \subset C^{n}\right.$ or $\left.S \subset R^{n}\right), S$ admits only a finite number of disjoint Zariski-open subsets.

\section{A strengthened form of the Rigidity Theorem.}

THEOREM 18.1. Let the base field of $E$ be $C$ (resp. R) and let $m \in M$ be such that $H^{1}\left(E, D_{m}\right)=0$. Then the orbit $G(m)$ is a Zariski-open subset of $M$ (resp. is one component of a Zariski-open subset of $M$ ).

Theorem 18.1 is an immediate consequence of Theorem 16.2 and Proposition 17.1.

Let $A_{k, n}$ be the subset of $x \in M$ for which $\operatorname{dim} Z^{n}(x) \leqq k$. An elementary argument shows that $A_{k, n}$ is a Zariski-open subset of $M$. A similar argument shows that $B_{k, n}=\left\{x \in M \mid \operatorname{dim} B^{n}(x) \geqq k\right\}$ is a Zariski-open subset of $M$. Let $U_{n}=\left\{x \in M \mid H^{n}\left(E, D_{x}\right)=0\right\}$; then $U_{n}$ is the union of the family $\left\{A_{k, n} \cap B_{k, n}\right\}_{k \in Z}$ of Zariski-open subsets and hence is a Zariski-open subset of $M$. 
Corollary 18.2. The Zariski-open subset $U_{1}$ of $x \in M$ for which $H^{1}\left(E, D_{x}\right)=0$ is the union of a finite number of orbits of $G$.

This follows immediately from Theorem 18.1.

19. Two geometric lemmas. Let $m \in M$, and let $E=B \oplus H \oplus C$ be a Hodge decomposition with respect to $m$. We set

$$
M^{\prime}=\left\{x \in L \mid \pi_{B} \cdot[x, x]=0\right\} .
$$

It is clear that $M \subset M^{\prime}$. We can consider $M^{\prime}$ as an approximation to $M$. The following lemma shows that, locally, $M^{\prime}$ is a manifold "lying above $Z^{1}$."

LEMMA 19.1. There exist an $N(m, L)$, an $N\left(Z^{1}\right)$ and an analytic mapping $\phi: N\left(Z^{1}\right) \rightarrow C^{1}$ such that

$$
N(m, L) \cap M^{\prime}=\left\{m+z+\phi(z) \mid z \in N\left(Z^{1}\right)\right\} .
$$

Proof. Let $F$ denote the polynomial mapping $Z^{1} \times C^{1} \rightarrow B^{2}$

$$
F(z, u)=D_{m} u+\frac{1}{2} \pi_{B} \cdot[z+u, z+u]
$$

then $F(0,0)=0$. The differential $d F_{(0,0)}$ maps $(0, u) \in Z^{1} \times C^{1}$ to $D_{m} u$; hence $u \rightarrow d F_{(0,0)}(0, u)$ is an isomorphism of $C^{1}$ onto $B^{2}$. By the implicit function theorem, the equation $F(z, u)=0$ can be "solved" for $u$ in a neighborhood of $(0,0)$; i.e. there exist neighborhoods $N\left(Z^{1}\right)$, $N\left(C^{1}\right)$ and an analytic map $\phi: N\left(Z^{1}\right) \rightarrow N\left(C^{1}\right)$ such that $(z, u) \in N\left(Z^{1}\right)$ $\times N\left(C^{1}\right)$ satisfies $F(z, u)=0$ if and only if $u=\phi(z)$. The observation that $m+z+u$ lies on $M^{\prime}$ if and only if $F(z, u)=0$ completes the proof. The equation $[x, x]=0$, for $x \in L$, is equivalent to the set of equations

$$
\pi_{B} \cdot[x, x]=0, \quad \pi_{H} \cdot[x, x]=0, \quad \pi_{C} \cdot[x, x]=0 .
$$

The following lemma shows that, in an $N(m, L)$, if $x$ satisfies the first two of these equations, it also satisfies the third.

LEMMA 19.2. There exists an $N(m, L)$ such that, if $x \in N(m, L)$ and if $\pi_{B} \cdot[x, x]=0$ and $\pi_{H} \cdot[x, x]=0$, then $[x, x]=0$.

Proof. Let $x=m+a \in L$, and assume that $\pi_{B} \cdot[x, x]=\pi_{H} \cdot[x, x]$ $=0$. Then we have, by the Jacobi identity,

$$
\begin{aligned}
0 & =[x,[x, x]]=\left[m+a, \pi_{B} \cdot[x, x]+\pi_{H} \cdot[x, x]+\pi_{C} \cdot[x, x]\right] \\
& =\left(D_{m}+\operatorname{ad}_{E} a\right) \cdot \pi_{C} \cdot[x, x] .
\end{aligned}
$$

For each $a \in E^{1}$ the map $D_{m}+\operatorname{ad}_{E} a$ sends $C^{n}$ linearly into $E^{n+1}$. When $a=0$, this map is an injection. Hence there is an $N\left(E^{1}\right)$ such that the 
restriction of $D_{m}+\operatorname{ad}_{E} a$ to $C^{2}$ is an injection whenever $a \in N\left(E^{1}\right)$. Hence, for $a \in N\left(E^{1}\right)$ the condition $\left(D_{m}+\operatorname{ad}_{E} a\right) \cdot \pi_{C} \cdot[x, x]=0$ implies $\pi_{c} \cdot[x, x]=0$. The neighborhood $N(m, L)=m+N\left(E^{1}\right)$ satisfies the condition of the lemma.

20. Kuranishi families. We use the notations of $\$ 19$.

Theorem 20.1. If $H^{2}\left(E, D_{m}\right)=0$, then $M^{\prime}$ is locally identical with $M$. A neighborhood of $m$ in $M$ can be parametrized by an $N\left(Z^{1}\right)$. The subset parametrized by $H^{1} \cap N\left(Z^{1}\right)$ contains a representative of each equivalence class.

The proofs of these statements are, respectively, immediate consequences of the Lemmas 19.2, 19.1 and 16.1.

If $H^{2}\left(E, D_{m}\right)$ does not vanish, the algebraic set $M$ may have a singular point at $m$. The structure of $M$ near $m$ is described by the set of zeros of an analytic map (called the obstruction map) $\Omega$ of an $N\left(H^{1}\right)$ into $H^{2}$.

Let $\phi: N\left(Z^{1}\right) \rightarrow C^{1}$ be as in Lemma 19.1; we then define $\Omega: N\left(Z^{1}\right)$ $\rightarrow H^{2}$ by

$$
\Omega(z)=\pi_{H} \cdot[z+\phi(z), z+\phi(z)]
$$

The set

$$
\Re=\left\{m+h+\phi(h) \mid h \in H^{1} \cap N\left(Z^{1}\right), \Omega(h)=0\right\}
$$

is called a Kuranishi family of deformations of $m$. The following theorem shows that $\mathfrak{K}$ is locally complete at $m$.

Theorem 20.3. Let $m \in M$, and let $E=B \oplus H \oplus C$ be a Hodge decomposition relative to $m$.

(a) There exist neighborhoods $N(m, M)$ and $N\left(Z^{1}\right)$, and analytic maps $\phi: N\left(Z^{1}\right) \rightarrow C^{1}$ and $\Omega: N\left(Z^{1}\right) \rightarrow H^{2}$, with $\phi(0)=0$ and $\Omega(0)=0$, such that

$$
N(m, M)=\left\{m+z+\phi(z) \mid z \in N\left(Z^{1}\right), \Omega(z)=0\right\} .
$$

(b) Let $\nVdash=\left\{m+h+\phi(h) \mid h \in H^{1} \cap N\left(Z^{1}\right), \Omega(h)=0\right\}$. Then there exist $N\left(C^{0}\right)$ and $N_{1}(m, M)$ such that the map $(k, a) \rightarrow \exp (a) \cdot k$ is an analytic homeomorphism of $\Re \times N\left(C^{0}\right)$ onto $N_{1}(m, M)$.

Proof. By Lemmas $16.1,19.1$ and 19.2 we may choose neighborhoods $N\left(Z^{1}\right), N\left(H^{1}\right), N\left(C^{0}\right), N(m, L)$ and $N_{1}(m, L)$, and an analytic map $\phi: N\left(Z^{1}\right) \rightarrow C^{1}$ such that the following conditions are satisfied:

$N(m, L)$ and $N_{1}(m, L)$ satisfy the conditions of Lemma 19.2;

The neighborhoods $N\left(Z^{1}\right), N(m, L)$ and the map $\phi$ satisfy the conditions of Lemma 19.1; 
$N\left(H^{1}\right)=H^{1} \cap N\left(Z^{1}\right)$;

The map $F:(a, h, u) \rightarrow \exp (a) \cdot(m+h+u)$ is an analytic homeomorphism of $N\left(C^{0}\right) \times N\left(H^{1}\right) \times H\left(C^{1}\right)$ onto $N_{1}(m, L)$.

Let $\Omega$ be the map defined by (20.2). Then, since $N(m, L)$ satisfies the conditions of Lemma 19.2, an element $x \in N(m, L)$ lies on $M$ if and only if $\pi_{B} \cdot[x, x]=0$ and $\pi_{H} \cdot[x, x]=0$. The first equation is satisfied if and only if $x=m+z+\phi(z)$, for $z \in N\left(Z^{1}\right)$; such an element $x$ satisfies the second equation if and only if

$$
\begin{aligned}
0 & =\pi_{H} \cdot[m+z+\phi(z), m+z+\phi(z)] \\
& =\pi_{H} \cdot\left(2 D_{m}(z+\phi(z))+[z+\phi(z), z+\phi(z)]\right)=\Omega(z) .
\end{aligned}
$$

The neighborhood $N(m, M)=M \cap N(m, L)$ satisfies the conclusion of $20.3(\mathrm{a})$.

For the proof of (b) we note that $F(a, h, u)=\exp (a) \cdot(m+h+u)$ lies on $M$ if and only if $F(0, h, u)=m+h+u$ does. Since $N_{1}(m, L)$ satisfies the conditions of Lemma 19.2, we see that $m+h+u$ lies on $M$ if and only if $u=\phi(h)$ and $\Omega(h)=0$, i.e. if and only if $m+h+u \in \mathcal{K}$. The conclusion of 20.3(b) follows easily.

We note that the choice of a Kuranishi family is not canonical. It depends on the Hodge decomposition of $E$ and on the choice of a (sufficiently small) $N\left(Z^{1}\right)$. The proof of Theorem 20.3 above shows, in effect, that a Kuranishi family is any set $M \cap\left(m+F^{1}\right) \cap N(m, L)$, where $F^{1}$ is any complementary subspace to $B^{1}$ in $E^{1}$ and where $N(m, L)$ is any sufficiently small neighborhood of $m$ in $L$; that is, locally a Kuranishi family is the intersection of $M$ with a linear variety through $m$ in $L$ which is transversal to the orbit $G(m)$ at $m$ and is of complementary dimension to $G(m)$ in $L$.

\section{§5. Deformation theorems For ALGebraic GRADED LIE ALGEBRAS}

21. Notation and terminology. Throughout $\$ 5 E=\bigoplus_{n \in z} E^{n}$ will be an algebraic graded Lie algebra over an algebraically closed field $K$; $G$ is the structure group of $E$ and $G_{0}$ the identity component of $G$. All finite-dimensional affine spaces over $K$ will be given the Zariski topology; subsets are given the induced topology. Otherwise, we shall follow the terminology introduced in 15.

Let $X$ be an irreducible subset of the finite-dimensional vector space $V$ over $K$. We denote by $A(X)$ the algebra of polynomial functions on $X$ (i.e. the restrictions to $X$ of the polynomial functions on $V)$. Since $X$ is irreducible, $A(X)$ is an integral domain; its quotient field is denoted $F$. We define $\operatorname{dim} X$ as the transcendence degree of $F$ over $K$. If $x \in X$, we define $T(X, x)$, the tangent space to $X$ at $x$, as 
follows: $y \in V$ is an element of $T(X, x)$ if and only if, for every polynomial function $P$ on $V$ which vanishes on $X$, the differential $d P_{x}$ of $P$ at $x$, vanishes on $y$. The tangent space $T(X, x)$ is a linear subspace of $V$ of dimension $\geqq \operatorname{dim} X$. The point $x$ is a simple point of $X$ if $\operatorname{dim} T(X, x)=\operatorname{dim} X$; it is known that the simple points of $X$ form a nonempty open subset of $X$. For further details we refer the reader to [3, pp. 177-184].

Let $X$ be a closed irreducible subset of a vector space $V$ and let $\phi$ be a morphism (i.e. a polynomial mapping) of $X$ into a vector space $W$. We denote by $d \phi_{x}$ the differential of $\phi$ at the point $x \in X$. It is a linear map of $T(X, x)$ into $W$.

\section{The Rigidity Theorem.}

THEOREM 22.1. Let $m \in M$ be such that $H^{1}\left(E, D_{m}\right)=0$. Then the orbit $G(x)$ is an open subset of $M$.

Proof. It is sufficient to show that $G_{0}(m)$ is an open subset of $M$. Since $G_{0}$ is irreducible, each irreducible component of $M$ is stable under the action of $G_{0}$. Let $M_{1}$ be the irreducible component of $M$ which contains $m$. It will suffice to show that $G_{0}(m)$ is an open subset of $M_{1}$. For, if this is the case, then $G_{0}(m)$ cannot meet any other irreducible component of $M$, and hence $G_{0}(m)$ is an open subset of $M$. Let $P$ denote the polynomial mapping $x \rightarrow[x, x]$ of $L$ into $E^{2}$. Then the differential $d P_{m}$ is just the map $2 D_{m}$. Since $P$ vanishes on $M_{1}$, it follows that $Z^{1}(m)$, the kernel of $d P_{m}$, is included in the tangent space $T\left(M_{1}, m\right)$.

It follows from the definition of an algebraic graded Lie algebra and of an admissible derivation that the differential of the representation $\rho^{\prime}$ of $G$ on $E$ is just the map $y \rightarrow \operatorname{ad}_{E} y$ of $E^{0}$ into $\mathrm{gl}^{0}(E)$. It follows from [2, p. 122, Corollaire 1] and [3, p. 192, Proposition 2] that the tangent space $T\left(G_{0}(m), m\right)$ contains the space $B^{1}(m)$ $=\left\{\operatorname{ad}_{E} y \cdot m \mid y \in E^{0}\right\}$. Since $G_{0}(m) \subset M_{1}$, we have $T\left(G_{0}(m), m\right)$ $C T\left(M_{1}, m\right)$. Thus

$$
B^{1}(m) \subset T\left(G_{0}(m), m\right) \subset T\left(M_{1}, m\right) \subset Z^{1}(m) .
$$

The condition $H^{1}\left(E, D_{m}\right)=0$ thus implies that $T\left(G_{0}(m), m\right)$ $=T\left(M_{1}, m\right)$. Let $s=\operatorname{dim} T\left(G_{0}(m), m\right)$. Since $G_{0}$ acts transitively on $G_{0}(m)$, every point of $G_{0}(m)$ is a simple point; hence $\operatorname{dim} G_{0}(m)=s$. But we have

$$
s=\operatorname{dim} G_{0}(m) \leqq \operatorname{dim} M_{1} \leqq \operatorname{dim} T\left(M_{1}, m\right)=s ;
$$

thus $\operatorname{dim} M_{1}=\operatorname{dim} G_{0}(m)$. This implies that $M_{1}$ is the closure of $G_{0}(m)$. (We note that up to this stage we have not used the closure 
of $K$; our argument so far is valid for an algebraic graded Lie algebra over an arbitrary infinite field.)

According to [3, p. 193, Proposition 3], the orbit $G_{0}(m)$ contains an open subset of its closure $M_{1}$. Since $G_{0}$ acts transitively on the orbit, it follows easily that $G_{0}(m)$ is an open subset of $M_{1}$.

Corollary 22.2. Let $U_{1}=\left\{x \in M \mid H^{1}\left(E, D_{x}\right)=0\right\}$. Then $U_{1}$ is an open subset of $M$, and $G$ admits only a finite number of orbits on $U_{1}$.

Proof. The fact that $U_{1}$ is open follows as in $\$ 18$. Theorem 22.1 implies that the intersection of $U_{1}$ with each irreducible component of $M$ can contain at most one orbit of $G$; thus there are only a finite number of orbits of $G$ on $U_{1}$.

23. Kuranishi families in the algebraic case. Let $m \in M$ and let $E=B \oplus H \oplus C$ be a Hodge decomposition of $E$ with respect to $m$. We set $M^{\prime}=\left\{x \in L \mid \pi_{B} \cdot[x, x]=0\right\}$. The following lemmas are the algebrogeometric analogues of Lemmas 19.1 and 19.2 of $\$ 4$.

Lemma 23.1. There is precisely one irreducible component $M_{1}^{\prime}$ of $M^{\prime}$ which contains $m$. Furthermore, $m$ is a simple point of $M_{1}^{\prime}$ and $T\left(M_{1}^{\prime}, m\right)=Z^{1}$.

Proof. This follows immediately from [17, p. 354, Proposition 1], and from [17, p. 74, Proposition 3], which allows us to pass from a universal domain to the algebraically closed field $K$.

Lemma 23.2. There exists an $N(m, L)$ such that, if $x \in N(m, L)$ and if $\pi_{B} \cdot[x, x]=0$ and $\pi_{H} \cdot[x, x]=0$, then $[x, x]=0$.

The proof is the same as that of Lemma 19.2 of $\S 4$.

TheOREM 23.3. Let $m \in M$ be such that $H^{2}\left(E, D_{m}\right)=0$. Then there is precisely one irreducible component $M_{1}$ of $M$ which contains $m$. Furthermore, $m$ is a simple point of $M_{1}$, and $T\left(M_{1}, m\right)=Z^{1}(m)$.

The proof follows immediately from the two preceding lemmas.

If $H^{2}\left(E, D_{m}\right) \neq 0$, then, as in the analytic case, the situation is more complicated.

Theorem 23.4. (a) Let $\Omega$ denote the map $x \rightarrow \pi_{H} \cdot[x, x]$ of $L$ into $H^{2}$. Let $S=\left\{x \in M^{\prime} \mid \Omega(x)=0\right\}$ and let $M^{\prime \prime}$ be the union of the irreducible components of $S$ which contain $m$. Let $M_{1}$ be the union of the irreducible components of $M$ which contain $m$. Then $M^{\prime \prime}=M_{1}$.

(b) Let $\kappa_{0}=\left\{x \in M^{\prime} \mid \pi_{B} \cdot(x-m)=0, \Omega(x)=0\right\}$, and let $\mathcal{K}$ be the union of the irreducible components of $\aleph_{0}$ which contain $m$. Then $\Re$ is included in $M$. Furthermore, $\pi$ is a locally complete family of deforma- 
tions of $m$ (i.e. the orbit $G(\mathcal{K})$ is a neighborhood of $m$ on $M$ ).

Before proceeding with the proof of 23.4, we discuss the geometric meaning. Let $\mathfrak{K}^{\prime}=\left\{x \in L \mid \pi_{B} \cdot(x-m)=0, \pi_{B} \cdot[x, x]=0\right\}$. Then essentially the same argument given in Lemma 23.1 shows that there is precisely one component $\mathcal{K}^{\prime \prime}$ of $\mathcal{K}^{\prime}$ containing $m$, that $m$ is a simple point of $\mathcal{K}^{\prime \prime}$, and that $T\left(\mathcal{K}^{\prime \prime}, m\right)=H^{1}$. Let $\Omega^{\prime}$ denote the restriction of $\Omega$ to $\mathcal{K}^{\prime \prime}$; then $\Omega^{\prime}$ maps $\mathcal{K}^{\prime \prime}$ into $H^{2}$. Theorem 23.4 says that the set $\varkappa_{0}$ of zeros of $\Omega^{\prime}$ is a locally complete family of deformations of $m$.

Proof. It follows from the definitions that $M$ is included in $M^{\prime \prime}$. Lemma 23.2 implies that there is an $N(m, L)$ such that $S \cap N(m, L)$ is included in $M$. It follows easily that $M^{\prime \prime}$ is included in $M$. This proves 23.4(a).

For the proof of $23.4(\mathrm{~b})$ we shall need the following technical lemma from algebraic geometry. We follow the terminology of [4].

Lemma 23.5. Let $\phi$ be a morphism of the affine variety $X$ into the affine space $W$. Let $x$ be a simple point of $X$ such that the differential $d \phi_{x}$ is a surjection. Then $\phi$ maps every neighborhood of $x$ in $X$ onto a neighborhood of $\phi(x)$ in $W$.

Proof. An elementary argument shows that the set $X_{1}$ of points $x^{\prime}$ of $X$ such that $d \phi_{x^{\prime}}$ is surjective is an open subset of $X$. It follows from [4, p. 42, Corollaire 1] that $\phi\left(X_{1}\right)$ contains an open subset of the closure $Z$ of $\phi(X)$; in particular, $\phi\left(X_{1}\right)$ contains a simple point of $Z$. This implies that $\operatorname{dim} Z=\operatorname{dim} W$, hence that $Z=W$. Thus $\phi$ is a dominant morphism. An elementary inductive argument using [17, p. 152, Theorem 6] shows that there is precisely one irreducible component of $\phi^{-1}(\phi(x))$ containing $x$, and that the dimension of this component is equal to $\operatorname{dim} X-\operatorname{dim} W$. The conclusion now follows from [4, p. 195, Proposition 3].

Let $\phi: G \times\left(H^{1}+C^{1}\right) \rightarrow L$ be defined by $\phi(g, u)=g \cdot(m+u)$. Let $e$ denote the identity of $G$. Then $d \phi_{(e, 0)}$ maps $E^{0}$ (the tangent space of $G_{0}$ at $e$ ) onto $B^{1}$ and induces the identity isomorphism on $H^{1}+C^{1}$; thus $d \phi_{(e, 0)}$ is surjective. Moreover, $(e, 0)$ is a simple point of $G \times\left(H^{1}+C^{1}\right)$. It follows from Lemma 23.5 that $\phi$ maps every neighborhood of $(e, 0)$ onto a neighborhood of $m$ on $L$. We note that $\phi(g, u) \in M$ if and only if $m+u \in M$. It follows easily from this remark and the preceding result that $G(\mathcal{K})$ is a neighborhood of $m$ on $M$.

24. Geometric remarks. In conclusion we discuss briefly the underlying algebro-geometric ideas of the proofs of the Rigidity Theorem and of Theorem 23.4 on the existence of Kuranishi families. An 
analogue of the Rigidity Theorem can be proved in the following general circumstances. Let $V$ be a finite-dimensional vector space over an algebraically closed field $K$, let $P_{1}, \cdots, P_{m}$ be polynomial functions on $V$, and let $g$ denote the ideal generated by $P_{1}, \ldots, P_{m}$ in the algebra $A(V)$ of all polynomial functions on $V$. Let $S$ denote the algebraic set of common zeros of $P_{1}, \cdots, P_{m}$. Let $G$ be an algebraic subgroup of $\mathrm{GL}(V)$ such that $g$ is stable under the action of $G$ on $A(V)$. It follows easily that $S$ is stable under the action of $G$. Denote by $g$ the Lie algebra of $G$. If $x \in S$ we define $Z^{1}(x)$ to be the intersection of the kernels of the differentials $\left(d P_{1}\right)_{x}, \cdots,\left(d P_{m}\right)_{x}$. We set $B^{1}(x)=\mathfrak{g}(x)$. It follows from [3, p. 192, Proposition 2] that $B^{1}(x)$ $C Z^{1}(x)$; we set $H^{1}(x)=Z^{1}(x) / B^{1}(x)$. (We note that the cohomology space $H^{1}(x)$ is not determined by the algebraic set $S$; however it is determined by the ideal $g$.) If $H^{1}(x)=0$, then the argument used in the proof of Theorem 22.1 shows that the orbit $G(x)$ is an open subset of $S$. If $K$ is either $R$ or $C$, it is sufficient to assume that $G$ is a Lie subgroup of $\mathrm{GL}(V)$. (In case $K=R$, we get a slightly weaker result, exactly analogous to Theorem 18.1 of $\S 4$.)

In a number of applications outlined in $\$ 3$ the cohomology spaces $H^{1}\left(E, D_{m}\right)$ can be explicitly computed. In the general situation we have discussed above, the computation of $H^{1}(x)$ may be quite involved.

The key geometric result in the proof of Theorem 23.4 is Lemma 23.2. Roughly speaking, this lemma says that in defining $M$ locally, (i.e. in a neighborhood of $m$ ), we can eliminate a certain number of the defining equations. To be more precise, let $n_{2}=\operatorname{dim} E^{2}$, let $b_{2}$ $=\operatorname{dim} B^{2}(m)$, and let $z_{2}=\operatorname{dim} Z^{2}(m)$. Then $M$ is defined as the set of common zeros of $n_{2}$ polynomials. It follows easily from dimensional considerations that at least $b_{2}$ polynomials are needed to define $M$ locally. Lemma 23.2 says that no more than $z_{2}$ equations are required to define $M$ locally. In particular, if $H^{2}(m)=0$, then $z_{2}=b_{2}$ and it follows readily that $m$ belongs to precisely one irreducible component of $M$ and is a simple point of that component. It turns out that a similar result holds for an arbitrary set of polynomial equations. In this case the cohomology defined by Harrison [10] seems to be the pertinent one. We plan to discuss this further in a future paper.

\section{REFERENCES}

1. C. Chevalley, Theory of Lie groups. I, Princeton Univ. Press, Princeton, N. J., 1946. 1951.

2. - Thêorie des groupes de Lie. II. Groupes algébriques, Hermann, Paris, 
3. - Thêrie des groupes de Lie. III. Théorèmes généraux sur les algèbres de Lie, Hermann, Paris, 1955.

4. - Fondements de la gémétrie algébrique, Course notes at the Institut Henri Pioncaré, Paris, 1958.

5. C. Chevalley and S. Eilenberg, Cohomology theory of Lie groups and Lie algebras, Trans. Amer. Math. Soc. 63 (1948), 85-124.

6. J. Dieudonne, Foundations of modern analysis, Academic Press, New York, 1960.

7. A. Frölicher and A. Nijenhuis, $A$ theorem on stability of complex structures, Proc. Nat. Acad. Sci. U.S.A. 43 (1957), 239-241.

8. M. Gerstenhaber, The cohomology structure of an associative ring, Ann. of Math. 78 (1963), 267-289.

9. - On the deformation of rings and algebras, Ann. of Math. 79 (1964), 59-104.

10. D. Harrison, Commutative algebras and cohomology, Trans. Amer. Math. Soc. 104 (1962), 191-204.

11. G. Hochschild, On the cohomology groups of an associative algebra, Ann. of Math. 46 (1945), 58-67.

12. K. Kodaira, L. Nirenberg and D. C. Spencer, On the existence of deformation of complex analytic structures, Ann. of Math. 68 (1958), 450-459.

13. M. Kuranishi, On the locally complete families of complex analytic structures, Ann. of Math. 75 (1962), 536-577.

14. - New proof for the existence of locally complete families of complex structures, Proc. Conf. Complex Analysis (Minnesota), Springer, Berlin, 1965; pp. 142154.

15. S. Lang, Introduction to differentiable manifolds, Interscience, New York, 1962.

16. A. Nijenhuis and R. W. Richardson, Jr., Cohomology and deformations of algebraic structures, Bull. Amer. Math. Soc. 70 (1964), 406-411.

17. A. Weil, Foundations of algebraic geometry, Amer. Math. Soc. Colloq. Publ. Vol. 29, rev. ed. Amer. Math. Soc., Providence, R. I., 1962.

18. - Introduction d l'étude des variêtes Kähleriennes, Hermann, Paris, 1958.

19. $\mathrm{H}$. Whitney, Elementary structure of real algebraic varieties, Ann. of Math. 66 (1957), 545-556.

University of Pennsylvania and

The Mathematical Institute, Oxford

University of Washington, Seattre 